\title{
Saccades Can Be Aimed at the Spatial Location of Targets Flashed During Pursuit
}

\author{
JOHN SCHLAG, MADELEINE SCHLAG-REY, AND PAUL DASSONVILLE \\ Department of Anatomy and Cell Biology and the Brain Research Institute, University of California \\ at Los Angeles Medical School, Los Angeles, California 90024
}

\section{SUMMARY AND CONCLUSIONS}

1. If an eccentric, stationary target is flashed while a subject is performing an eye movement in the dark, can this subject make a saccade to the location in space where the target briefly appeared? Different predictions result from alternative hypotheses regarding the manner in which saccade goals are determined. Retinal error being defined as the vector from the eye position at the time of the flash to the position of the target, the retinal-error hypothesis predicts that the saccade vector will be equal to the retinal-error vector. This hypothesis assumes that the oculomotor system ignores the eye displacement between target presentation and saccade. If so, the target will be missed. In contrast, the spatial-error hypothesis predicts that the eye displacement is taken into account by the brain to calculate the target's physical location to which, therefore, a correct saccade could be aimed.

2. At issue is the generality of a fundamental principle of ocular targeting. Previous studies have established that, if the movement is saccadic, eye displacement is used by the oculomotor system to calculate the target's physical location. In the case of pursuit, perceptual experiments on humans suggest that eye displacement is taken into account although its velocity is underestimated. However, in a recent study McKenzie and Lisberger reported that saccade trajectories starting during pursuit conform to the retinal error hypothesis. In other words, velocity underestimation is close to $100 \%$.

3. Although McKenzie and Lisberger's results are very clear, they might have depended on particular cxperimental conditions. The issue was reinvestigated in a situation facilitating the discrimination of stimuli. In the present study monkeys were trained, separately, to make saccades to briefly flashed targets and to pursue linearly moving ones as long as they were visible. The two tasks were then combined. While monkeys pursued a first target, a second was flashed. The animals tracked the first target, extinguished after $600-800 \mathrm{~ms}$, and the smooth eye movement continued for another 130-300 ms before the saccade occurred. The objective of the experiment was to determine whether the saccade would reach the spatial (physical) location where the flash appeared.

4. Saccade trajectories were measured in three monkeys and compared with the trajectories predicted by the two hypotheses mentioned above. Actual trajectories were intermediate between the predicted ones, in accordance with the view that tracking velocity was underestimated. Two monkeys were intensively trained on the task. Statistical analysis of their data revealed that the direction of the saccade was closer to the one predicted by the spatial-error hypothesis, in contrast to McKenzie and Lisberger's results.

5. Because these authors used a shorter flash duration $(10 \mathrm{~ms}$ vs. $26 \mathrm{~ms}$ in our paradigm), tests were run in a third monkey with flashes of 5,30 , and $100 \mathrm{~ms}$, and the results were compared. As flash duration increased, saccade direction was found to shift away from retinal-error prediction toward spatial-error prediction.
6. Our results suggest that the accuracy of saccades to a stationary flash presented during pursuit depends on several factors, not all well understood. Because evidence is offered that such saccades can be aimed at the spatial location of the flashed target, models discounting the role of extraretinal signals in this condition appear unsupported.

\section{INTRODUCTION}

In 1976 Hallett and Lightstone did an experiment in which human subjects were required to look successively at two sites where photic stimuli had been consecutively flashed in total darkness. Humans can perform this task readily. Because the second saccade (from site A to site B) started from an eye position other than that at which the image of flash B had been impressed on the retina, that saccade could not have been correctly aimed using only the distance and direction of target B provided by its retinal image (i.e., retinal-error signal). The mechanism of target acquisition is not that simple. Hallett and Lightstone's finding suggests that voluntary saccades are directed to the spatial location of objects, a process that takes into account a neural signal indicating the direction of the line of sight. This conclusion agrees with the intuitive notion that we form internal representations of our environment and that we use internal maps to orient ourselves (Thomson 1980).

Hallett and Lightstone's results are verifiable whether the eye displacement intervening between flash presentation and its acquisition is a movement to a target (as in their original paradigm), a movement of search in the absence of any actual target (Schlag et al. 1989), or a movement evoked by electrical stimulation (Sparks and Mays 1983). In each of these cases the perturbing eye displacement is a saccade. However, the story might be different if the displacement is smooth as, typically, in visual pursuit. A recent study by McKenzie and Lisberger (1986) suggests that slow eye displacement is practically not taken into account. Presenting a brief flash to monkeys pursuing another target, these authors found that the saccade to the flash was directed to its retinal location. They argued that the saccade generator does not use extraretinal feedback about smooth changes in eye position, and they proposed a model eliminating the computation of spatial error during pursuit.

The remarkable finding in McKenzie and Lisberger's observations (1986) is that monkeys were aiming at targets as if their eyes had not moved at all. It is known that perceptual localization during pursuit is less accurate than after saccades (Miller 1980). When movements are slow, 
there is evidence that eye velocity is underestimated (Miller 1980), and eye position may not be continuously monitored. However, underestimation of perceived velocity depends on the experimental situation. It varies from $\sim 10$ (Mack and Herman 1972) to almost 100\% (Sedwick and Festinger 1976).

The paradigm used by McKenzie and Lisberger was originally designed to study perceptual localization (e.g., Ward 1976), but it offers interesting possibilities to explore visuooculomotor behavior. Monkeys are trained to pursue a horizontally moving target. At some unpredictable time, the moving target is extinguished and another one is flashed for $10 \mathrm{~ms}$ at one of several positions above (e.g., $6^{\circ}$ ) the eye trajectory. Because pursuit continues for a few hundred milliseconds after the extinction of the moving target, the eye position at the start of the saccade differs from the eye position at the time the second target was flashed. This distance, a function of saccade latency and eye velocity, plays an important role in distinguishing two hypotheses. The retinal-error hypothesis predicts that the saccade vector will be equal to the retinal-error vector, thus missing the target in space. The spatial-error hypothesis predicts that the saccade vector will be directed to the physical location of the flash, thus compensating for the smooth change in eye position. With pursuit at $30^{\circ} / \mathrm{s}$, McKenzie and Lisberger (1986) found a better statistical fit of actual saccade trajectories with the courses predicted by the retinal-error hypothesis.

What does this result mean? Let us note that monkeys in this situation never received visual feedback on where the second target actually was. Tracking in the dark is known to be a source of illusions because of the uncertainty regarding what moves and how much. Here the difficulty is compounded by the fact that the flashed target is visible for such a short time. Is the flash seen as a second, stationary target? Or is it interpreted as the first target, still moving horizontally, but suddenly jumping upward and then disappearing? The first interpretation calls for a saccade to the spatial location of the flash; the second one, to the site where the flash was assumed to have moved, which is indicated by the retinal-error vector transposed at saccadc onset. As the monkey was rewarded in either case for making a saccade, it had no cue favoring one interpretation over the other. What was tested under these conditions is the animal's own preference in an ambiguous situation rather than the ability of its oculomotor apparatus to perform a given process like computing a spatial-error signal.

Has it been demonstrated that saccades initiated during pursuit cannot be directed to the spatial location of a target? In fact, McKenzie and Lisberger (1986) already answered this question in a particular case. One of their monkeys, which had earlier practice in which the flashed target was relit, developed a consistent bias toward spatial error. Although the target had always been presented at the same place, this animal had to calculate its location from ever-changing eye positions. But even in the absence of visual feedback, it is hard to imagine that smooth eye displacement would be totally ignored in aiming a saccade to a target briefly seen while pursuing another one. Such a view would be discordant with the conclusions of most perceptual studies (e.g., Sedwick and Festinger 1976), all the more so that oculomotor accuracy is in general better than perceptual judgements (Miller 1980). We thought that the problem should be reinvestigated under conditions in which the discrimination of stimuli and the performance of the task were made easier.

For this purpose, some quantitative changes were made in the original paradigm. A delay (up to $800 \mathrm{~ms}$ ) was introduced between the extinctions of the flashed target and of the moving onc. Monkeys were trained to keep tracking as long as the pursuit stimulus was visible; otherwise the trial was aborted. This change brought several advantages. First, it made it clearer that there were not one but two distinct stimuli because both were displayed concurrently. Second, the excursion of the smooth eye displacement could be increased several times (e.g., $15^{\circ}$ or more vs. $4-6^{\circ}$ in McKenzie and Lisberger's experiment), thereby widening the gap between the results predicted by the two hypotheses and improving the resolving power of the test. Third, longer flashes could be used, which theoretically facilitates target localization and the perception of target stationarity. Fourth, the velocity of the moving target could be lowered, which rendered pursuit easier and more precisc. Conversely, one drawback of postponing the saccade might be that the accuracy of aiming at a remembered location deteriorates, as has been shown in the first $500 \mathrm{~ms}$ of waiting (Gnadt et al. 1990).

In two monkeys, the data obtained under these conditions are compared with those reported by McKenzie and Lisberger (1986). In a third monkey, we tested the influence of changing flash duration. These results are presented here as evidence bearing on the current controversy opposing retinal and spatial models of saccade generation.

\section{METHODS}

Under deep pentobarbital sodium anesthesia, three monkeys (Macaca nemestrina) were operated to place a coil around one eyeball and to build a cement pedestal over the skull to attach and restrain the head. Eye-position signals were recorded by the magnetic search-coil method.

Monkcys sat in a primate chair facing a tangent screen at a distance of $132 \mathrm{~cm}$. The screen subtended a visual angle of $61^{\circ}$ horizontally and $50^{\circ}$ vertically. In total darkness photic stimuli $\left(0.23^{\circ}\right.$-diam spots $)$ were back-projected through a wide-angle lens from a 608 Tektronix oscilloscope equipped with a P11 short persistence tube. Stimulus presentation was controlled by computer.

All three animals were initially trained to make saccades to targets that could appear at any time and at any place on the screen. Thereafter, they learned two preparatory tasks. In the first one they were periodically rewarded for pursuing a moving target continuously until its extinction. Eye position had to remain within a $12^{\circ}$-electronic window centered on the target. Linear target velocity was progressively increased to $28^{\circ} / \mathrm{s}$. In the second task, monkeys had to keep fixating a steady point while a second target was bricfly projectcd elsewhere. They were rewarded by a drop of grape juice for making a correct saccade to the second target location after the fixation point had been turned off. The fixation point was relit at the same position $500 \mathrm{~ms}$ later to allow for correction of aiming. The exposure duration of the second target was gradually reduced from 500 to $26 \mathrm{~ms}$, whereas the delay up to extinction of the fixation point was lengthened to $1 \mathrm{~s}$. The criterion of targeting was also made more stringent by reducing to $8^{\circ}$ the size of the electronic window around the flashed target.

After this initial phase of practice with pursuit and target ac- 
quisition, the experimental schedules diverged as follows. The first two monkeys $J$ and $V$ were intensively trained (daily for 3 and $3 \frac{1}{1} 2 \mathrm{wk}$, respectively) on the experimental task, and data were collected for comparison with those obtained by McKenzie and Lisberger (1986). The objective was different with the third monkey Z: it was to test the effect of changing flash duration. This monkey had no prior practice with the task itself when data collection started. Data were obtained in individual sessions interspersed in a way to minimize the transfer of experience or habit between sessions (see below).

The experimental task, which combined features of the two preparatory tasks, is illustrated in Fig. 1 with the usc of an actual record from monkey $Z$. The stable fixation point was replaced by a target moving horizontally at velocities from 16 to $28 \%$ s (pursuit was leftward in the trial shown in Fig. 1). The flash occurred at unpredictable times during pursuit but at least $400 \mathrm{~ms}$ after the eye had entered the electronic window centered on the moving target. This target was extinguished $600-800 \mathrm{~ms}$ later (the delay was kept constant for all trials within a session). The position of the flashed target (marked T in Fig. 1) was varied horizontally in a pseudorandom manner with equal numbers of right, center, and left presentations in equal numbers of rightward and leftward pursuits. The vertical position was held constant throughout individual sessions at $6^{\circ}$ below or $1.5-20^{\circ}$ above the pursuit trajectory. The reason for using different levels was to prevent monkeys from learning that any stereotyped eye movements, for instance upward, would be rewarded throughout all sessions. An important point is that the flashed target was never relit during the pursuit task. Therefore monkeys were never cued regarding their aiming performance. As in the original McKenzie and I isberger study, the electronic window was extended horizontally so that saccades were rewarded whenever they terminated within $4^{\circ}$ of the vertical position of the flashed target. Initially, saccades tended to overshoot the targets upward by $3-8^{\circ}$, as noted by others (Sparks and Mays 1983) when saccades are executed to flashes in the dark. This overshoot could be only partly corrected by reintroducing practice trials with a steady point of fixation and relighting of the target. Saccades also tended to be curved, which

A

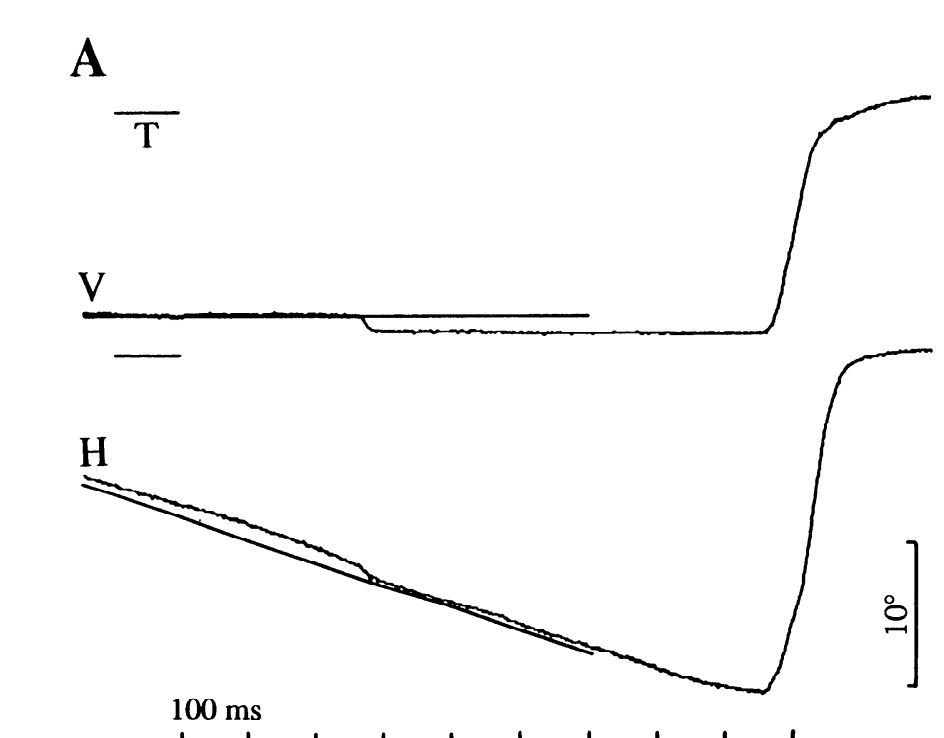

$100 \mathrm{~ms}$ is common when their trajectory is oblique (Viviani et al. 1977), especially if they are aimed at a remembered location in darkness (Gnadt et al. 1990; Smit and Van Gisbergen 1990).

With the first two monkeys, the second target was flashed always for $26 \mathrm{~ms}$ (instead of $10 \mathrm{~ms}$ as in McKenzie and Lisberger 1986). The third monkey was tested in pairs of sessions with flash durations of 5 and $30 \mathrm{~ms}$ on three consecutive days, but the order was alternated: $5-30 \mathrm{~ms}$ on day $1,30-5 \mathrm{~ms}$ on day 2 , and again 5-30 ms on day 3. On day 15, a session was run with steady flashes of $100-\mathrm{ms}$ duration and, on day 44 , two consecutive sessions: one with moving, the other with steady flashes, both of 100-ms duration. Training was continued between experimental sessions as in the preliminary phase (see above).

To avoid fatigue, trials were separated by several seconds; and their number (range: 105-290) was limited in individual sessions. Horizontal and vertical signals of eye and stimuli were recorded on polygraph paper and on analog magnetic tape for off-line display on a CRT screen and digitized (1-kHz sampling rate) on computer. Direction and distance errors between actual saccade endpoints and the terminations predicted by the spatial-error and retinal-error hypotheses were measured by computer. In Fig. 1B, the retinal-error vector is shown by the dashed line from the eye position at the offset of the flashed target to the position of that target $(T)$. The goal predicted by the retinal-error hypothesis $\left(E_{r}\right)$ is the same vector transposed at saccade onset. The goal predicted by the spatial-error hypothesis is the physical location of $\mathrm{T}$, close to which the actual saccade ended in this particular trial. As can be seen in Fig. $1 B$, the distance between the two hypothetical goals $(\nearrow$ ) is a function of length of pursuit (including small vertical eye shifts as in this case) plus saccade reaction time $(130-300 \mathrm{~ms})$. Usually, this distance corresponded to $\sim 15^{\circ}$.

\section{RESULTS}

\section{Saccades to a flashed target in two monkeys intensively trained on a pursuit task}

For the sake of comparison, the results of the first two monkeys $J$ and $V$ were analyzed and are presented in simi-

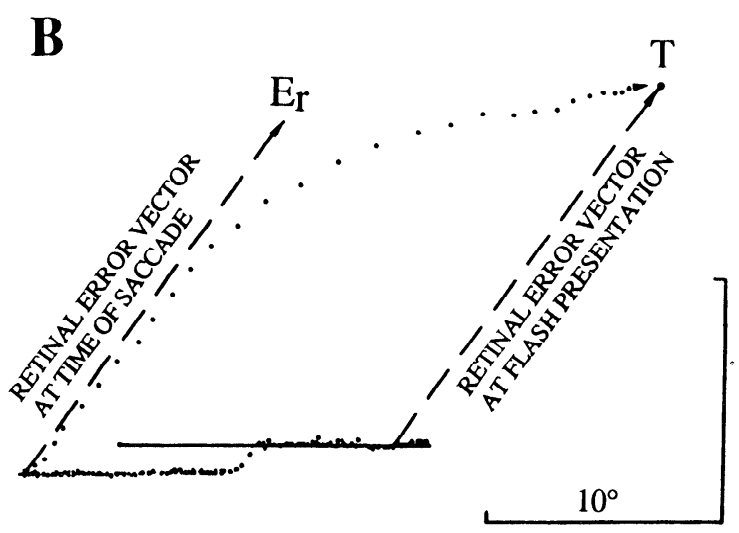

FIG. 1. Experimental paradigm illustrated by records of 1 trial in monkey $Z$. A: vertical (V) and horizontal (H) tracings of eye and stimuli versus time. $B$ : vertical versus horizontal display of eye and stimulus positions of same trial. Eye trajectory shown by dotted line (sampling rate for illustration, $250 \mathrm{~Hz}$; sampling rate for data analysis was higher, $1 \mathrm{KHz}$ ). First target was moving leftward at $15.3^{\circ} / \mathrm{s}$ (therefore record $B$ should be read from right to left). Second target was presented at site (T) and was turned off $600 \mathrm{~ms}$ before the end of the pursuit target. Saccade latency, $252 \mathrm{~ms}$. Dashed lines in $B$ indicate retinal-error vector plotted twice: at flash presentation, and transposed at time of saccade toward point $\mathrm{E}_{r}$, which is the termination predicted by retinal-error signal. Spatial error hypothesis predicts that saccade termınates at site $\mathrm{T}$. 


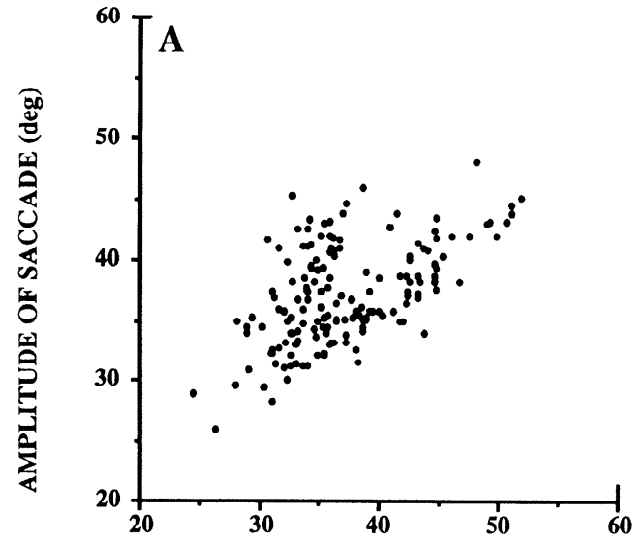

AMPLITUDE OF RETINAL ERROR

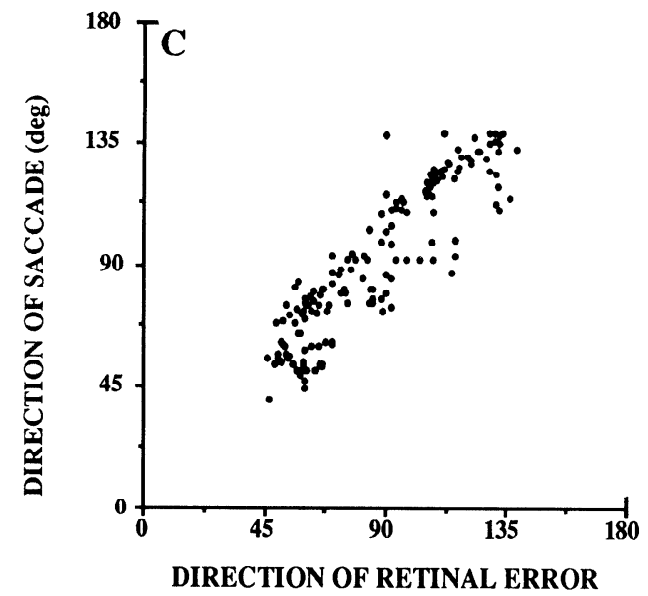

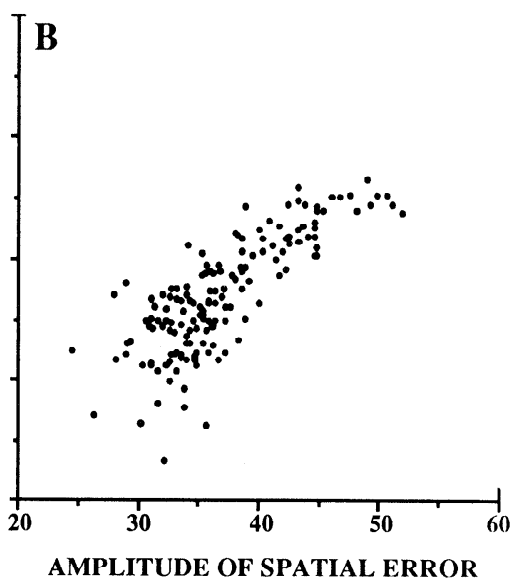

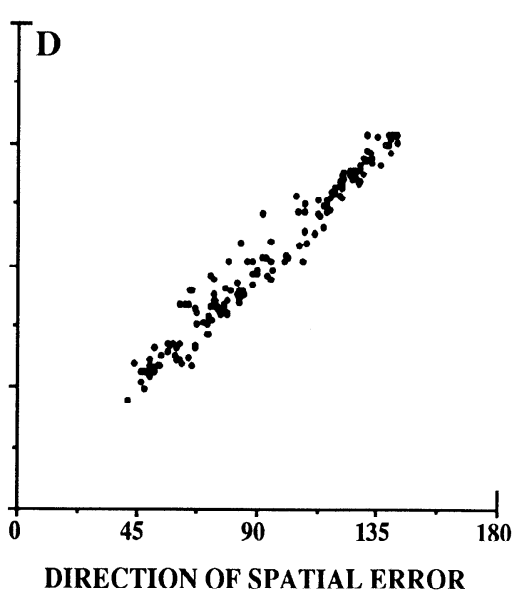

FIG. 2. Scattcr plot showing relations of actual saccade vectors with retinal-error predictions (left panels) and spatial-error predictions (right panels). Top panels compare vector amplitudes. Bottom panels compare vector directions. Direction is expressed in degrees. Zero degree is a leftward vector and $180^{\circ}$ is rightward. Data from monkey $\mathrm{V}$ at day $10(n=152)$. Pursuit velocity, $16^{\circ} / \mathrm{s}$; delay, $600 \mathrm{~ms}$; flash duration, $26 \mathrm{~ms}$. lar form as in McKenzie and Lisberger's study (1986). Throughout successive sessions, actual saccade directions toward the sites of flashed targets were intermediate between the courses predicted by the retinal- and spatial-error hypotheses (see below and Fig. 4). However, in contrast to McKenzie and Lisberger's data (1986), ours showed a better fit with the spatial-error prediction. This observation applies to every session, and it is supported by different quantitative analyses.

As a typical example, Fig. 2 illustrates data from one monkey after nine days of practice with the experimental task. The amplitude (top panels) and direction (bottom panels) of actual saccades are plotted against the values predicted on the basis of the retinal and spatial hypotheses. Each plotted point represents a single saccade. Points would line up along a diagonal line if the fit were perfect. The lesser scatter apparent in the right panels strongly suggests a better correlation of the observed saccades with those predicted by the spatial-error hypothesis. This was verified by calculating the coefficients of correlation between the parameters of actual and predicted saccade trajectories. They are given for one day in middle training and for the last day of recording of both monkeys.

Although the coefficients of correlation varied appreciably, those related to spatial errors were always higher than those related to retinal errors. A $t$ test of significance of difference between dependent correlations (Bruning and
Kintz 1968) was applied to these data. The values are given in Table 1 under each of the eight pairs compared (amplitude and direction on 2 different days in 2 monkeys). The values found significant at $P<0.001$ are marked by asterisks.

As McKenzie and Lisberger did, we replotted the direction data of Fig. 2 (bottom panels) in a manner that graphically reveals the relative importance of deviations from

TABLF 1. Correlation of saccade parameters with retinal and spatial error

\begin{tabular}{lcccc}
\hline \hline & \multicolumn{4}{c}{ Monkey } \\
\cline { 2 - 5 } & $J I$ & $J 2$ & $V I$ & $V 2$ \\
\hline $\begin{array}{l}\text { Saccade vs. spatial-error } \\
\quad \text { direction }\end{array}$ & 0.883 & 0.958 & 0.980 & 0.925 \\
$\begin{array}{l}\text { Saccade vs. retinal-error } \\
\quad \text { direction }\end{array}$ & 0.332 & 0.704 & 0.905 & 0.790 \\
$\begin{array}{l}t \text { test of significance of } \\
\text { difference }\end{array}$ & $14.06^{*}$ & $19.73^{*}$ & $11.27^{*}$ & $5.75^{*}$ \\
$\begin{array}{l}\text { Saccade vs. spatial-error } \\
\text { amplitude }\end{array}$ & 0.551 & 0.946 & 0.806 & 0.630 \\
$\begin{array}{c}\text { Saccade vs. retinal-error } \\
\text { amplitude }\end{array}$ & 0.514 & 0.611 & 0.428 & 0.455 \\
$t$ test of significance of \\
difference
\end{tabular}

$* P<0.001$. 
retinal and spatial errors (top panel of Fig. 3). The bottom panel shows the results of the same analysis on the measurements made on the last day in the other monkey. This is the case in which variability was greatest. In Fig. 3 the difference between actual- and spatial-error directions is plotted on the ordinate against the difference between retinal- and spatial-error directions on the abscissa. In this mode of representation, datum points should align on a horizontal line if the data are correlated with the spatialerror hypothesis, and the slope of the linear regression line should be 0 . Conversely, the points should collect on the diagonal line if the retinal-error hypothesis is favored, and the slope of the linear regression line should be 1 . The actual slopes were, respectively, 0.161 and 0.232 in these cases, and 0.189 for all data collected in the two monkeys.

Most points in the top panel of Fig. 3 are located between the horizontal and diagonal lines, as if saccade trajectories

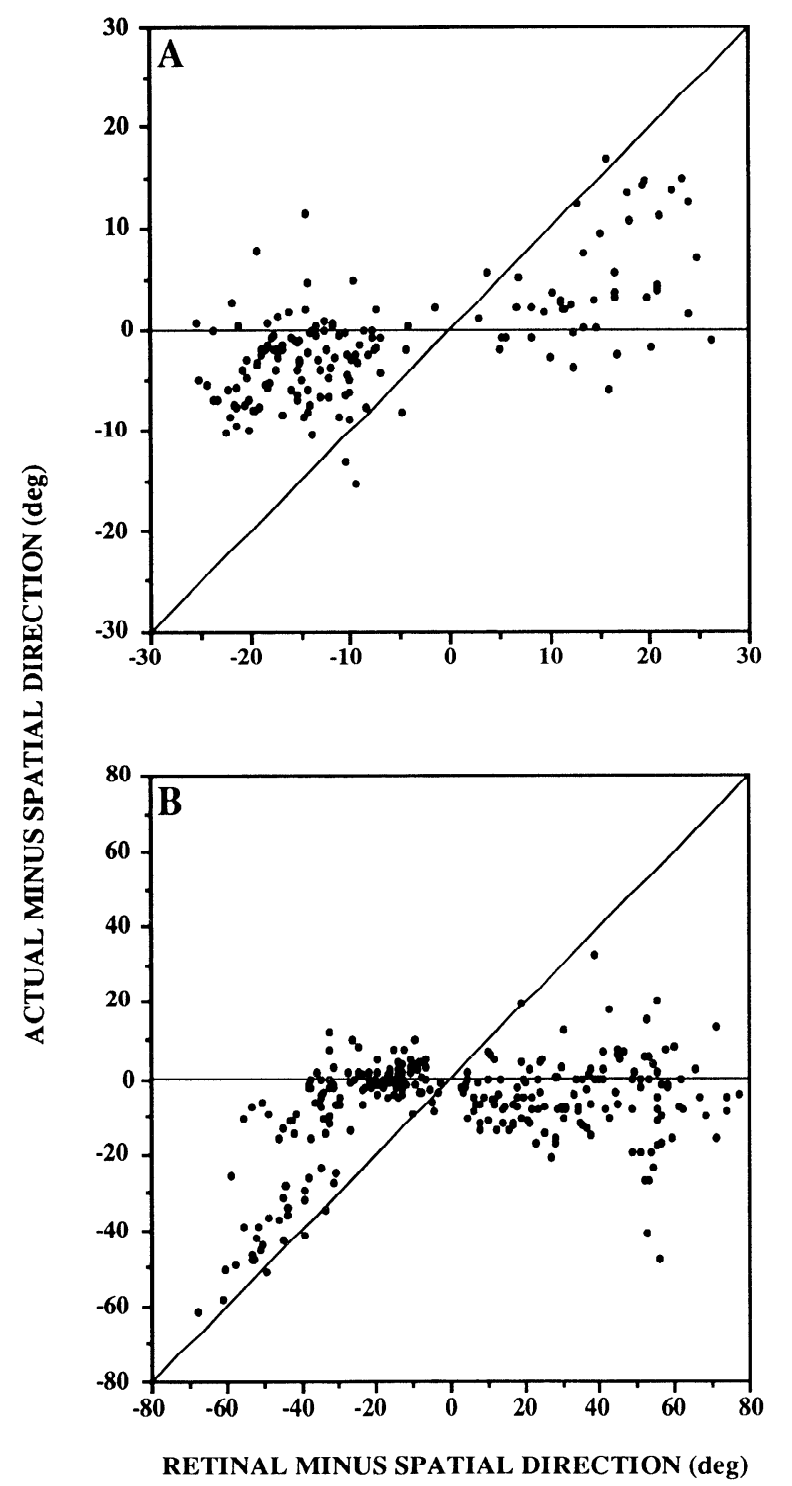

FIG. 3. Regression analysis comparing relative deviations of saccade directions from directions predicted by retinal and spatial error hypotheses. $A$ : same data as in Fig. 2. B: data from monkey $J$ on last day of recording $(n=290)$. Pursuit velocity, $20^{\circ} \%$; delay, $800 \mathrm{~ms}$. were influenced by both retinal and spatial errors. This trend was apparent throughout successive sessions. In a few sessions (as illustrated in the bottom panel), relatively separate groupings of points were noticeable along both lines, as if the animals were using the retinal error at some times and the spatial error at other times. Preferences could be biased in relation with the direction of pursuit (e.g., preference for spatial error with rightward pursuit and for retinal error with leftward pursuit, or the reverse, which lasted for 5-10 trials). However, this was far from consistent. Alternations could occur even in successive trials that were comparable in direction of pursuit, stimulus position, and timing.

In four sessions ( 2 in each monkey), the flashed targets were presented very low $\left(1.5^{\circ}\right)$ above the pursuit trajectory so that the vertical components of saccades were minimal. On 146 trials selected because the directions of predicted saccades were opposite (right vs. left), 131 were oriented in agreement with spatial error, whereas 15 were in agreement with retinal error.

\section{Effect of flash duration on saccade aiming}

We hypothetized that discrepancies between our results with the first two monkeys $J$ and $V$ and the results of McKenzie and Lisberger (1986) could be due to individual differences among subjects, to flash duration, or both. Because the effect of flash duration was amenable to testing, data were collected with three duration values in monkey $Z$.

As the principal objective here is a comparison between these data rather than with those of McKenzie and Lisberger (1986), we prefer to use a mode of presentation supplying more complete information on where actual saccades terminated with respect to predicted endpoints. Figure 4 displays the horizontal positions of saccade terminations on a percentage scale where $0 \%$ corresponds to the retinal-error prediction and $100 \%$ to the spatial-error prediction. The whole scale represents $\sim 15^{\circ}$, the exact value depending on saccade latencies in individual trials. Normalized distributions are shown for flash durations of $5 \mathrm{~ms}$ in $A$ (results pooled from 3 sessions), $30 \mathrm{~ms}$ in $B$ ( 3 sessions), and $100 \mathrm{~ms}$ in $C 1$ ( 2 sessions) and $C 2$ ( 1 session). Panel $D$, which reproduces the data of monkey $V$ in Fig. 2, is provided here as a reference to relate the present results with those of the first two monkeys.

It can be verified that the large majority of saccades terminated at intermediate positions between those predicted by the hypotheses under consideration, a fact already mentioned. With flash durations of 5 and $30 \mathrm{~ms}$, the performance of monkey $Z$ resembled more closely that of McKenzie and Lisberger's animals (10-ms flashes) than that of our first two monkeys (26-ms flashes). Whatever its cause, variability among subjects must be recognized as real.

In monkey $Z$, a difference is noticeable between the distributions of panels $A$ and $B$. With 5-ms flashes, $77.3 \%$ of the results conformed better with the retinal-error hypothesis (panel $A$ ) versus $61.1 \%$ with 30 -ms flashes (panel $B$ ). With 100-ms flashes, this percentage fell to 27.5 (panel C1). This effect of flash duration was consistent even when individual sessions were compared. 


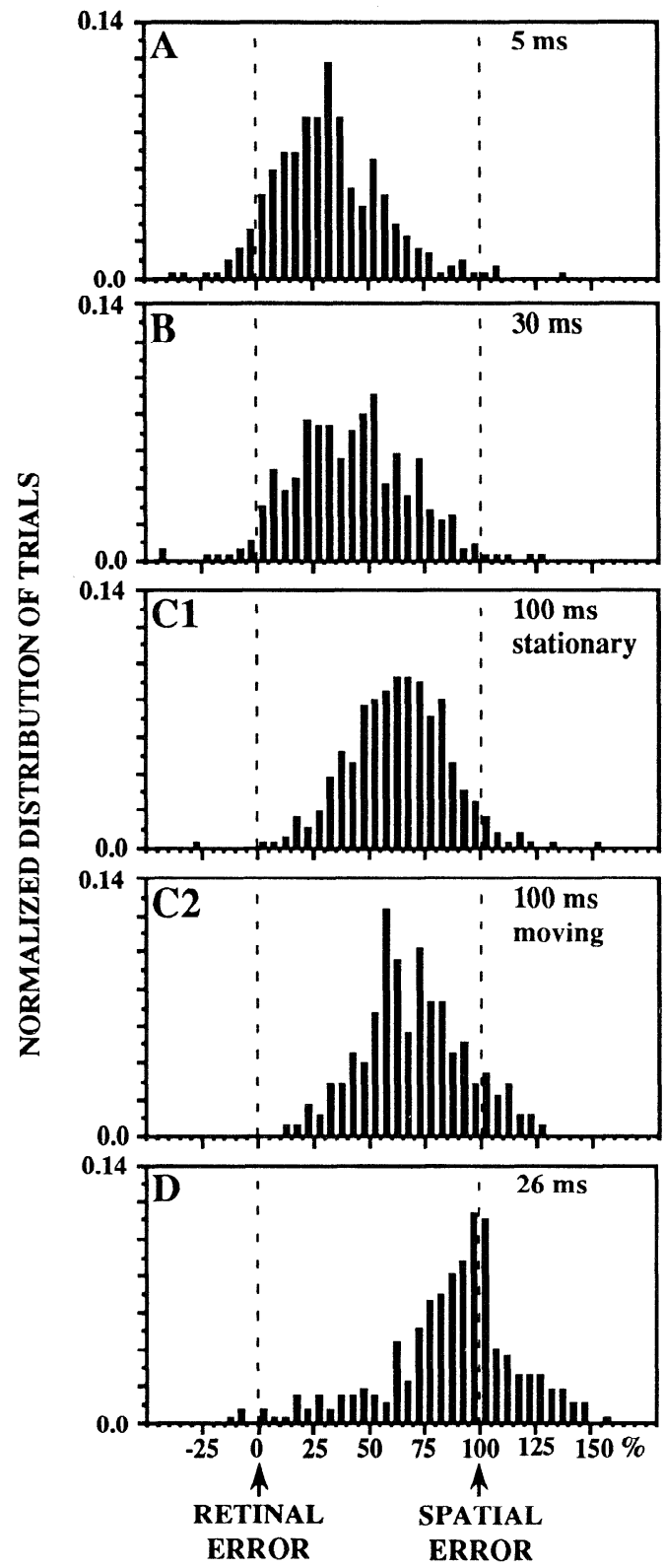

FIG. 4. Normalized distributions of deviations of saccade directions from directions predicted by retinal and spatial error hypotheses. On abscissa, $0 \%$ corresponds to retinal error and $100 \%$ to spatial error. $A-C 2$ : data from monkey $Z$ with different flash durations. $A$ : $5 \mathrm{~ms}(n=318) . B$ : $30 \mathrm{~ms}(n=370)$. $C 1: 100$-ms stationary flash $(n=359)$. $C 2: 100-\mathrm{ms}$ moving flash $(n=179)$. $D$ : same data $(26 \mathrm{~ms})$ as in Fig. 2 provided for reference.

In the first session of day 44 , the flashed target was moving (for $100 \mathrm{~ms}$ ) parallel to and at the same velocity as the pursuit target. The objective was to determine whether the monkey would take this cue into account to assume that the whole display was moving. If so, it should rely on the retinal error alone to program its saccades. This did not happen (panel C2). On that session, only $17.9 \%$ of the saccades were better predicted by the retinal-error hypothesis.

\section{DISCUSSION}

Several reasons can be advanced for the apparent discrepancy between McKenzie and Lisberger's observations (1986) and ours. The simplest explanation invokes differ- ences among animals, possibly related to previous experience (e.g., our monkeys were never rotated in a chair). But even though flashes of comparable durations were used (30 vs. $26 \mathrm{~ms}$ ), our monkey $Z$ did not perform as did the first two. Its results were closer to those reported by McKenzie and Lisberger. There were even fluctuations in the way each animal tended to aim at the target within a session. Some variations might have been due to a wandering level of attention while executing a very rapid, repetitive, task.

Sccond, whereas data in both studies have been analyzed in terms of the retinal-spatial alternative, a third option needs to be considered. It is a variant of the spatial-error hypothesis, relevant particularly when the interval between target flashing and saccade onset is very short as in McKenzie and Lisberger's study (1986). Because the eyes are moving and because the delay of transmission of retinal signals to the brain is long, the eye position at the time a flash image becomes available to the brain to calculate a spatial error is no longer what it was at flash presentation (Mateeff et al. 1981). One usually assumes that the oculomotor system still has access to the initial eye-position value. But there are indications that this assumption is probably wrong (Ward 1976). One possible reason is that eye position may not be continuously sampled when eye movements are smooth. Then the brain may have to use the current eye position, i.e., read when the retinal-error signal arrives. If so, the resulting saccade will aim (incorrectly) at some intermediate position between those predicted by the straightforward retinal- and spatial-error hypotheses. Some of McKenzie and Lisberger's records may be explained in this manner. For instance, the records of their Figs. 1 and 2 are compatible with the hypothesis that spatial error was calculated from the eye position achieved some $100 \mathrm{~ms}$ after target presentation. Because this time is a nonnegligible fraction of the saccade latency (sometimes as short as $130 \mathrm{~ms}$, see their Fig. 2), the actual saccade goal appears closer to the one predicted by the retinal-error hypothesis than by the spatial-error hypothesis. Our results may have been similarly affected, however much less. Indeed, the difference obtained by using one or the other eye positions as reference to estimate a spatial error (i.e., the distance travelled during $100 \mathrm{~ms}$ or less of pursuit) and the variations around this difference (Ward 1976) become negligible when the saccade is postponed by $800 \mathrm{~ms}$ or more.

Third, the lower velocity of pursuit and the longer duration of the second target in our experimental task were probably determinant. With monkey $Z$, evidence was obtained that at least the second of these factors dramatically affected the results. Theoretically, a longer time of exposure should make a stimulus brighter and more salient. But it can also blurr the image or make it appear as a streak of light when the eyes move in front (Holly 1975). For instance, during saccades Matin et al. (1972) have shown the smear to be maximum for flash durations around 20-40 $\mathrm{ms}$. It is not clear whether target localization might have been facilitated and, if so, why? However, in animal $/$, aiming was directed preferably toward the spatial location of the target when it moved at the pursuit velocity (see Fig. $4, C 2)$. In this condition, the image should be relatively stable on the retina. Human subjects perceive a stimulus as an immobile spot when it travels with, and at about the same speed as, an eye movement. We are not aware of any 
similar experiment of localization during pursuit. Regarding saccades, one study (Holly 1975) has indicated that the spot appears located at the start, another one (Mateeff 1978) along the midcourse of the trajectory, not at the end where the retinal-error hypothesis would place it.

More complete investigations are obviously needed to assess the importance of various factors determining saccade decisions during pursuit. Nlthough the effect of lengthening flash duration may not be explained, the important point is the result achieved by this procedure. We do not contest McKenzie and Lisberger's observations (1986), but we question the general conclusion these authors derived from experiments performed under limited conditions. Our evidence indicates that eye displacement during pursuit can be taken into account to determine the physical location of briefly presented targets. This conclusion seems inescapable even though eye velocity was often grossly underestimated because saccades usually terminated between the goals predicted by the retinal and spatial hypotheses. Our conclusion is also in agreement with many studies on visual perception during pursuit (Festinger and Canon 1965; Mack and Herman 1978; Miller 1980; Sedwick and Festinger 1976).

McKenzie and Lisberger (1986) recognized the need of flexibility in targeting mechanisms, so that aiming will be adapted to the subject's interpretation of the visual display. They argued that, if the flashed target and the pursued one are perceived as moving together, the spatial error is of no use. Therefore an eye-position signal is unnecessary to calculate target location in space. The retinal-error signal suffices. But the only circumstance in which these authors considered that the flashed target is seen as stationary is when it remains visible until the saccade is programmed. The authors correctly remarked that computing a spatial error is irrelevant also in this case. The retinal error can indeed provide an estimate of target coordinates that is not too far off the mark. However, this argument does not apply to our study because the targets were turned off several hundred milliseconds before saccade onset. Nevertheless, monkeys' gaze landed close to the spatial location of these targets. This finding suggests that the flexibility of saccade mechanisms envisioned by McKenzie and Lisberger (1986) cannot be achieved by the use of retinal-error signals only. Current models are probably too simplistic, but, at least, they should include an estimate of eye position to be summed with retinal error as circumstances dictatc.

This work was supported by United States Public Health Service Grants EY-02305 and EY-05879 and by National Science Foundation Fellowship RCD87-58034.
Address for reprint requests: J. Schlag, Dept. of Anatomy, University of California-Los Angeles Medical School, Los Angeles, CA 90024-1763.

Received 1 November 1989; accepted in final form 9 April 1990.

\section{REFERENCES}

BRUNing, J. L. AND KINTZ, B. L. Computational Handbook of Statistics. Glenview, IL: Scott, Foresman, 1968, p. 193.

FESTINGER, L. AND CANON, L. K. Information about spatial location based on knowledge about efference. Psychol. Rev. 72: 373-384, 1965.

Gnadt, J. W., Bracewell, R. M., AND ANDERSEn, R. A. Sensorimotor transformation during eye movements to remembered visual targets. Vision Res. In press.

Hallett, P. E. And Lightstone, A. D. Saccadic eye movements towards stimuli triggered by prior saccades. Vision Res. 16: 99-106, 1976.

Holly, F. Saccadic presentation of a moving target. Vision Res. 15: 331-335, 1975.

HONDA, H. Perceptual localization of visual stimuli flashed during saccades. Percept. Psychophys. 45: 162-174, 1989.

MACK, A. AND HERMAN, E. A new illusion: the underestimation of distance during pursuit eye movements. Percept. Psychophys. 12: 471-473, 1972.

Mack, A. AND Herman, E. The loss of position constancy during pursuit eyc movements. Vision Res. 18: 55-62, 1978.

MATEEFF, S. Saccadic eye movements and localization of visual stimuli. Percept. Psychophys. 24: 215-224, 1978.

MateefF, S., YakimofF, N., AND Dimitrov, G. Localization of brief visual stimuli during pursuit eye movements. Acta Psychol. 48: 133-140, 1981.

MAtin, E., Clymer, A., AND MATin, L. Metacontrast and saccadic suppression. Science Wash. DC 178: 179-182, 1972.

Mckenzie, A. And Lisberger, S. G. Properties of signals that determine the amplitude and direction of saccadic eye movements in monkeys. $I$. Neurophysiol. 56: 196-207, 1986.

MILLER, J. M. Information used by the perceptual and oculomotor systems regarding the amplitude of saccadic and pursuit eye movements. Vision Res. 20: 59-68, 1980.

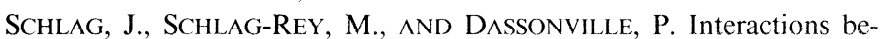
tween natural and electrically evoked saccades. II. At what time is eye position sampled as a reference for the localization of a target? Exp. Brain Res. 76: 548-558, 1989.

SEDWICK, H. A. AND FESTINGER, L. Eye movements, efference, and visual perception. In: Eye Movements and Psychological Processes, edited by R. A. Monty and J. W. Senders. Hillsdale, NJ: Erlbaum, 1976, p. $221-230$

Smit, A. C. AND VAN Gisbergen, J. A. M. An analysis of curvature in fast and slow human saccades. Exp. Brain Res. In press.

SPARKS, D. L. AND MAYS, L. E. Spatial localization of saccade targets. I. Compensation for stimulus induced perturbations in eye position. I. Neurophysiol. 49: 45-74, 1983.

Viviani, P., Berthoz, A., AND Tracey, D. The curvature of oblique saccades. Vision Res. 17: 661-664, 1977.

Thomson, J. A. How do we use visual information to control locomotion? Trends Neurosci. 3: 247-250, 1980.

WARD, F. Pursuit eye movements and visual localization. In: Eye Movements and Psychological Processes, edited by R. A. Monty and J. W. Senders. Hillsdale, NJ: Erlbaum, 1976, p. 289-297. 